\title{
Understanding and Treatment of Peripheral Neurotoxicity Induced by Chemotherapy
}

\author{
Yang Zhou ${ }^{1}$, Yami Zhang ${ }^{2 *}$ \\ ${ }^{1}$ Shaanxi University of Chinese Medicine, Xianyang 712000, China \\ ${ }^{2}$ Affiliated Hospital of Shaanxi University of Chinese Medicine, Xianyang 712000, China
}

\begin{abstract}
This paper analysis chemotherapy-induced peripheral neuropathy (CIPN) caused by related chemotherapeutic drugs and proposes some treatments from nursing intervention to drug treatment. It also calls for more efforts in the research of this field to relieve psychological burden and improve quality of life of patients at the end of the paper.
\end{abstract}

Key words: CIPN; Neurotoxicity; Treatment

Publication date: May, 2021; Publication online: 31 May, 2021

*Corresponding author: Yami Zhang, 451312201@qq.com

In recent years, the age of cancer onset has a trend of low age, and the gender difference is shrinking, which seriously affects human health and life expectancy. As a traditional anti-tumor method, chemotherapy cannot accurately identify tumor cells and kill normal cells at the same time. In the process of treatment, it often produces a series of toxic and side effects. With the application of platinum, taxanes, alkaloids, fluorouracil and other chemotherapy drugs, chemotherapy-induced peripheral neuropathy (CIPN) has gradually become a common clinical side effect, affecting the use of chemotherapy drugs and clinical efficacy, affecting the quality of life of patients, and even forced to terminate chemotherapy. Western medicine for CIPN mainly includes calcium and magnesium, sodium channel blockers, vitamins, etc., which can relieve symptoms to a certain extent, but the effect is not satisfactory.

\section{CIPN caused by related chemotherapeutic}

\section{drugs}

Cisplatin and Oxaliplatin are the main platinum compounds that cause CIPN. Cisplatin (DDP) is widely used in clinic. Its anti-tumor mechanism is to inhibit DNA replication and transcription, inhibit tumor cell proliferation and induce tumor cell apoptosis ${ }^{[2]}$. Oxaliplatin (OXA) is the third-generation platinum anticancer drug, which is commonly used in the treatment of digestive system malignant tumors. The antitumor spectrum is similar to cisplatin, and there is no cross resistance between them, but it has stronger cytotoxicity, and the incidence of peripheral neurotoxicity is as high as $85 \%-95 \%{ }^{[3]}$.

\subsection{Peripheral neurotoxicity induced by cisplatin}

Studies have shown that the higher the cumulative dose of cisplatin is, the higher the incidence of peripheral neurotoxicity is. When the cumulative dose reaches $300 \mathrm{mg} /$ $\mathrm{m}^{2}, 45 \%$ of the patients have peripheral neurotoxicity, and when the cumulative dose reaches $500-600 \mathrm{mg} / \mathrm{m}^{2}$, almost $100 \%$ of the patients have peripheral neurotoxicity symptoms. This study also points out that the peripheral neurotoxicity caused by cisplatin is mostly reversible and recovers in more than 15 weeks $^{[4]}$.

\subsection{Oxaliplatin induced peripheral neurotoxicity}

Oxaliplatin induced peripheral neurotoxicity is related to the cumulative dose. According to its clinical manifestations, it can be divided into two categories. One is acute neurotoxicity, which can occur within a few hours or 1-2 days after administration, and generally lasts for no more than 7 days. It is often induced or aggravated by cold stimulation. The main manifestations are peripheral nerve paralysis or defect, such as mild finger (toe) end or perioral paresthesia, dullness or tenderness. The periodic use of 
drugs may cause repeated symptoms. There are also patients with acute laryngeal spasm, but it is very rare. The second is chronic neurotoxicity, with slow onset and long course of disease. In the early stage, it is mainly manifested as numbness and paresthesia. In the later stage, it may appear sensory loss, accompanied by ataxia and dysfunction, and finally progress to physical dysfunction ${ }^{[5]}$.

\subsection{Taxanes induced peripheral neurotoxicity}

Paclitaxel induced peripheral neurotoxicity generally occurs within 3 days after medication, about $30.1 \%$ of patients have grade I-II neurotoxicity, and $10 \%$ of patients have grade III-IV neurotoxicity ${ }^{[6]}$. The typical clinical manifestations are glove sock numbness and burning sensation at the end of limbs. A small number of patients have decreased vibration and disappeared deep tendon reflex. In severe cases, symptoms such as sensory loss and tremor paralysis may appear at the distal end of limbs, showing symmetry ${ }^{[7]}$.

\section{Treatment of peripheral neurotoxicity caused}

\section{by chemotherapy}

\subsection{General methods and nursing intervention}

As OXA induced peripheral neurotoxicity is often induced or aggravated by cold stimulation, patients should be advised to keep warm before and after chemotherapy and avoid contact with low-temperature articles and cold air. At the same time, the time of intravenous infusion should be controlled to avoid the highest concentration of drugs in plasma. Patients with acute neurotoxicity should be controlled at 2-3 hours, and patients with chronic neurotoxicity should be prolonged to 6 hours $^{[8]}$. For chronic neurotoxicity caused by OXA, the "stop and go" strategy of intermittent use of Oxaliplatin is currently used in clinic to improve the threshold of cumulative dose of neurotoxicity. In the route of administration, changing the route of administration can reduce the peripheral nerve injury of chemotherapy drugs and reduce the incidence of CIPN.

\subsection{Drug treatment}

At present, there is no unified standard for the treatment of CIPN in western medicine, mainly drug treatment, including calcium and magnesium, antioxidant and so on.

\subsubsection{Total calcium and magnesium}

Calcium and magnesium are commonly used in the prevention and treatment of CIPN, which can avoid or reduce the effect of OXA on neural membrane channels. Sodium channels can be changed by calcium ions, and then sodium influx increases. Magnesium sulfate can reduce the stress of nerve fibers, restore the function of ATPase, and facilitate the operation of sodium pump.

\subsubsection{Sodium channel blockers}

The acute neurotoxicity induced by OXA is closely related to the transient changes of sodium channels. The toxic and side effects of OXA can be alleviated by sodium channel blockers, which has been confirmed by many studies.

\subsubsection{Antioxidants}

Antioxidants are another research direction of CIPN control, including reduced glutathione (GSH), amifostine (Amifostine for injection) and $\alpha$ - lipoic acid

GSH can promote the formation of low toxic compounds which are easy to metabolize and reduce the toxicity of some exogenous poisons. GSH is a neuromodulator, known as scavenger of free radicals, which can prevent platinum drugs from accumulating on DRG and has a protective effect on neurons ${ }^{[10]}$.

\section{Conclusion}

Chemotherapy induced peripheral neurotoxicity (CIPN) is worthy of our attention. Mild CIPN results in increased psychological burden and decreased quality of life of patients, and severe CIPN results in chemotherapy pause, which seriously affects the treatment process of malignant tumor. We expect more researchers to invest in CIPN and get better treatment plan, so as to reduce the psychological and physiological burden of patients with malignant tumor and continue the chemotherapy plan.

\section{References}

[1] Zou XL, Chen P, Xie M, et al. Progress in treatment of chemotherapy-induced peripheral neurotoxicity with traditional Chinese and Western Medicine [J]. Hunan Journal of traditional Chinese Medicine, 2018, 34 (3): 200-202.

[2] Wang $\mathrm{T}$ H, Wan J Y, Gong X, et al. Tetrandrine enhances cytotoxicity of cisplatin in human drug-resistant esophageal squamous carcinoma cells by inhibition of multidrug resistance-associated protein $[\mathrm{J}]$. Oncology Reports, 2012, 28(5): 1681-6.

[3] Ma F, Yuan P, Xu Q, Xu BH. Clinical analysis and treatment of acute neurotoxicity induced by Oxaliplatin [J]. Journal of Clinical Oncology, 2005(5): 533-535.

[4] Troy L, Mcfarland K, Littmanpower S, et al. Cispl antin-based therapy: a neurological and neuropsycho 
logical review [J]. Psycho-oncology, 2000, 9(1): 29.

[5] Chen XB, Lv HF, Chen BB, Luo SX. Research pr ogress on mechanism and prevention ofOxaliplatin Neurotoxicity [J]. Chinese Medical Science, 2012, 2(3): 38-40.

[6] Noguchi Y, Nishimura R, Kawara H, et al. Survey of current status of adverse ocular reactions to pa clitaxel and aretrospective analysis for aiding in ear ly detection of adversereactions [J]. Gan to Kagaku Ryoho Cancer \& Chemotherapy, 2013, 40(6): 81922.

[7] Kiya T, Kawamata T, Namiki A, et al. Role of sat ellite cell-derived L-serine in the dorsal root gangli on in paclitaxel-induced painful peripheral neuropat hy [J]. Neuroscience, 2001, 174(2): 190.

[8] Krishnan AV, Goldstein D, Friedlander M, et al. Oxaliplatin-induced neurotoxicity and the development of neuropathy [J]. Muscle \& Nerve, 2005, 32(1): 51.

[9] Gong CX. Clinical observation on Yiqi Tongluo Decoction in treating chemotherapy-induced peripheral neurotoxicity [D]. Shandong University of traditional Chinese Medicine, 2010.

[10] Catalano V, Cascinu S, Labianca R, et al. Neuroprotective effect of giutathione(GSH) on Oxaliplatin (L-OHP)-based chemotherapy in advanced colorectal cancer patients(pts): a randomized double-blind placebo-controlled trial [J]. European Journal of Cancer, 2001, 37(Suppl 6): S280-S282. 